\title{
Petatewalide B alleviates oxygen-glucose deprivation/reoxygenation-induced neuronal injury via activation of the AMPK/Nrf2 signaling pathway
}

\author{
SUN YOUNG PARK ${ }^{1}$, MIN HYUN CHO ${ }^{2}$, MEI LI $^{2}, \mathrm{KE} \mathrm{LI}^{2}$, GEUNTAE PARK $^{3}$ and YOUNG-WHAN CHOI ${ }^{2}$ \\ ${ }^{1}$ Bio-IT Fusion Technology Research Institute, Pusan National University, Busan 46241; \\ ${ }^{2}$ Department of Horticultural Bioscience, Pusan National University, Miryang, Gyeongsangnam 50463; \\ ${ }^{3}$ Department of Nanomaterials Engineering, Pusan National University, Busan 46241, Republic of Korea
}

Received October 8, 2019; Accepted April 1, 2020

DOI: $10.3892 / \mathrm{mmr} .2020 .11075$

\begin{abstract}
Neuronal injury is a common, and critical, occurrence in clinical ischemic strokes, and can cause irreversible brain damage. However, the precise pathological mechanisms underlying this condition and effective treatment remain unclear. Increasing evidence shows that the nuclear factor erythroid 2-related factor 2 (Nrf2)/activated protein kinase (AMPK) signaling pathway serves a significant role in neuronal injury and is involved in neuroprotection. The present study demonstrated that petatewalide $B$, the active constituent of Petasites japonicus, otherwise known as butterbur, can alleviate oxygen-glucose deprivation/reoxygenation (OGD/R)-induced neuronal death via the adenosine monophosphate-AMPK/glycogen synthase kinase (GSK)-3/ $\beta / \mathrm{Nrf} 2 /$ antioxidant response element (ARE) signaling pathways in human neuroblastoma SH-SY5Y cells. A neuronal injury model was established by depriving SH-SY5Y cells of oxygen and glucose for $8 \mathrm{~h}$, followed by $24 \mathrm{~h}$ of reoxygenation (OGD/R). The results indicated that the OGD/R model exhibited reduced cell viability but increased lactate dehydrogenase (LDH) release, reactive oxygen species (ROS) production and apoptosis. These were accompanied by increased levels of cleaved PARP, cleaved caspase-9, cleaved caspase-3, p53, Bax and p21, as well as decreased Bcl-2 levels.
\end{abstract}

Correspondence to: Professor Young-Whan Choi, Department of Horticultural Bioscience, Pusan National University, 1268-50 Samnangjin-ro, Miryang, Gyeongsangnam 50463, Republic of Korea

E-mail: ywchoi@pusan.ac.kr

Professor Sun Young Park, Bio-IT Fusion Technology Research Institute, Pusan National University, 2 Busandaehak-ro 63beon-gil, Geumjeong-gu, Busan 46241, Republic of Korea

E-mail: sundeng99@pusan.ac.kr

Key words: petatewalide B, Petasites japonicus, oxygen-glucose deprivation/reoxygenation, neuroprotection, 5' AMP-activated protein kinase, nuclear factor erythroid 2-related factor 2
Treatment with petatewalide B was able to strengthen cell viability but reduced LDH release, ROS production and the expression levels of apoptosis-related proteins. Additionally, treatment with petatewalide B activated AMPK in the OGD/R-exposed SH-SY5Y cells and upregulated activation of the downstream transcription factor $\mathrm{Nrf} 2$, which accompanied heme oxygenase 1 (HO-1) and $\mathrm{NAD}(\mathrm{P}) \mathrm{H}$ quinone dehydrogenase 1 (NQO1) expression. Furthermore, silencing AMPK, Nrf2, HO-1 and NQO1 expression inhibited petatewalide B's protective effect against apoptosis in the OGD/R-exposed SH-SY5Y cells. Therefore, petatewalide B protected human neuroblastoma cells against OGD/R-induced injury by downregulating apoptosis and oxidative stress via upregulation of the AMPK/Nrf2 signaling pathway, suggesting that petatewalide $\mathrm{B}$ may be a prospective protector against neuronal injury, having possible therapeutic and medical implications.

\section{Introduction}

The brain is the most easily injured organ of the human body when ischemia/reperfusion (I/R) occurs. I/R-induced injury is defined as the process in which tissue injury occurs during initial hypoxia, followed by oxygen supply recovery. This common injury may be caused by organ transplantation, resection, and other clinical conditions, including iatrogenic processes (1). Although the underlying pathogenesis of I/R injury is not fully understood, studies have shown that oxidative stress may be involved. Oxidative stress is frequently the result accumulated reactive oxygen species (ROS) and is defined as an imbalance between the production and elimination of free radicals (2). Studies revealed that oxidative stress may cause secondary injury to existing organ injuries by promoting apoptosis, suggesting it has an important role in therapies for neuronal injuries (3). The body developed various antioxidant mechanisms to counterbalance oxidative stress, such as the nuclear factor erythroid 2-related factor 2 (Nrf2)/antioxidant response element (ARE) signaling pathway.

Nrf2 is a critical transcription factor responsible for alleviating cellular oxidative stress and protecting cells from oxidative stress damage. When exposed to stressors, Nrf2 dissociates from its endogenous suppressor, Kelch-like epoxy 
chloropropane-associated protein-1 (Keap1). Subsequently, the Nrf2 nuclear localization signal induces rapid translocation to the nucleus for activation (4). Upon entry into the nucleus, Nrf2 binds to the ARE signaling pathway and initiates the transcription of cytoprotective and antioxidant genes, thereby promoting cell survival. Among these genes, heme oxygenase 1 (HO-1) and $\mathrm{NAD}(\mathrm{P}) \mathrm{H}$ quinone dehydrogenase 1 (NQO1) represent essential endogenous antioxidants and cytoprotective enzymes. During the onset of I/R-induced injury, HO-1 and NQO1 expression in neuronal cells increases. In addition, several studies have found that the Nrf2/ARE signaling pathway can inhibit neurotoxicity by limiting oxidative stress activation and suppressing the apoptosis signaling pathway $(5,6)$. Therefore, therapeutic strategies for regulating antioxidant capacity and neuroprotective properties may be effective in treating neuronal injury.

Adenosine monophosphate-activated protein kinase (AMPK) exerts a crucial role in brain protection regulation and is involved in neuronal injury in I/R. Recent data suggest that AMPK does not play an exclusive role in the $I / R$ injury response; rather, it is an endogenous defensive molecule that mediates neuroprotective effects in $I / R$ injury $(7,8)$. A previous study reported that activation of the AMPK signaling pathway inhibits abnormal oxidative stress, thereby reducing ROS production and exerting neuroprotective effects. Previous research suggests that AMPK induces neuroprotection of neuronal cells by targeting Nrf2/ARE. AMPK may also indirectly regulate Nrf2 activation via glycogen synthase kinase (GSK)-3 $\beta$ phosphorylation $(9,10)$, which induces the ARE-mediated neuroprotective response. Furthermore, the AMPK/Nrf2 signaling pathway was reported to mediate reducing effects on $I / R$ injury in neuronal cells $(10,11)$.

Recently, phytochemicals that can elicit improvements in neurodegenerative diseases have received extensive attention. Many phytochemicals can play valuable roles in preventing and treating neurodegenerative diseases because of their strong antioxidant properties (12). P. japonicus has many pharmacologically active constituents, including petatewalide $\mathrm{B}$, kaempferol, peteasitin, and quercetin. Petatewalide B is a novel sesquiterpene compound and is the most important active constituent of $P$. japonicus $(13,14)$. Furthermore, sesquiterpenoids from $P$. japonicus play a key role in aging-related disorders, such as atherosclerosis, hyperlipidemia, Parkinson's disease, Alzheimer's disease, and memory disorders $(15,16)$. However, petatewalide B's neuroprotective properties and mechanisms have not been fully elucidated. In this study, we attempted to explore petatewalide B's potential neuroprotective mechanisms in SH-SY5Y cells exposed to OGD/R.

\section{Materials and methods}

Cell culture and establishment of the cell model of I/R injury. The human-derived neuroblastoma SH-SY5Y cells were obtained from the American Type Culture Collection and maintained in Dulbecco's modified Eagle's medium (DMEM) (Gibco; Thermo Fisher Scientific, Inc.), supplemented with 10\% heat-inactivated fetal bovine serum and $1 \%$ penicillin/streptomycin (Invitrogen; Thermo Fisher Scientific, Inc.), under a humidified atmosphere $\left(95 \%\right.$ air, $5 \% \mathrm{CO}_{2}$ ) at $37^{\circ} \mathrm{C}$. The I/R injury cell model was used to perform oxygen and glucose deprivation/reoxygenation (OGD/R). The cells were washed twice with phosphate-buffered saline (PBS) and then cultured with OGD medium (glucose-free and serum-free DMEM) under hypoxic conditions $\left(1 \% \mathrm{O}_{2}, 95 \% \mathrm{~N} 2\right.$, and $\left.5 \% \mathrm{CO}_{2}\right)$ for $8 \mathrm{~h}$ at $37^{\circ} \mathrm{C}$, followed by quick reoxygenation $(95 \%$ air and $5 \% \mathrm{CO}_{2}$ ). The medium was substituted with regular medium for $24 \mathrm{~h}$. Petatewalide B was purified from $P$. japonicus leaves as previously described in another study (13). Petatewalide B was solubilized with dimethyl sulfoxide (DMSO) and pretreated with petatewalide B (5-40 $\mu \mathrm{M})$ for $1 \mathrm{~h}$ before OGD/R treatment.

MTT and lactate dehydrogenase (LDH) assay. The 3-(4,5-dimethylthiazol-2-yl)-2,5-diphenyltetrazolium bromide (MTT) and LDH assays were performed to detect the treated SH-SY5Y cells' viability and cytotoxicity. SH-SY5Y cells were seeded into 24 -well plates at the density of $1 \times 10^{5}$ cells $/ \mathrm{ml}$. The SH-SY5Y cells were treated with petatewalide B (5-40 $\mu \mathrm{M})$ or OGD/R after $24 \mathrm{~h}$ of culture. In brief, cells were incubated with MTT reagent $(50 \mu \mathrm{g} / \mathrm{ml})$ and maintained for a further $6 \mathrm{~h}$. Thereafter, cells were treated with DMSO for $5 \mathrm{~min}$. Each sample was analyzed using a microplate reader (Perkin-Elmer) at $570 \mathrm{~nm}$. The LDH assay was performed using the cytotoxicity detection kit (LDH; Roche Applied Science), according to the manufacturer's instructions. Readings were recorded using a microplate reader (Perkin-Elmer) at an absorbance of $490 \mathrm{~nm}$.

Measurement of intracellular ROS. The treated cells' intracellular ROS levels were evaluated using the ROS assay kit (CM- $\mathrm{H}_{2}$ DCFDA; Thermo Fisher Scientific, Inc.) according to manufacturer's instructions. In brief, SH-SY5Y cells were seeded into 6-well plates at a density of $1 \times 10^{5}$ cells $/ \mathrm{ml}$. After $24 \mathrm{~h}$ of culture, the SH-SY5Y cells were treated with petatewalide B $(20 \mu \mathrm{M})$ or OGD/R. The SH-SY5Y cells were rinsed with PBS and incubated with $\mathrm{CM}-\mathrm{H}_{2} \mathrm{DCFDA}$ for $30 \mathrm{~min}$ in the dark. The fluorescent intensity was proportional to the intracellular ROS levels. Thereafter, intracellular ROS levels were determined according to the fluorescent intensity, which was recorded using a Flow Cytometer (Beckman Coulter FC500; Beckman Coulter).

Apoptosis assay. Flow cytometric analysis was conducted to investigate apoptosis in the treated cells. The SH-SY5Y cells were collected by trypsinization and washed thrice with PBS. Subsequently, cells were stained with Alexa Fluor ${ }^{\circledR} 488$ dye-labeled anti-BrdU antibody (APO-BrdU ${ }^{\mathrm{TM}}$ TUNEL assay kit; Invitrogen; Thermo Fisher Scientific, Inc.), according to the manufacturer's protocol. Finally, the cells were subjected to apoptosis analysis using the Flow Cytometer Cytomics FC 500 (Beckman Coulter).

Preparation of protein extracts. Total protein was extracted from cells using a RIPA Lysis and Extraction Buffer (Thermo Fisher Scientific, Inc.) supplemented with protease and phosphatase inhibitors (Roche). The nuclear extraction of SH-SY5Y cells was carried out using Thermo Scientific ${ }^{\mathrm{TM}}$ NE-PER ${ }^{\mathrm{TM}}$ Nuclear and Cytoplasmic Extraction kit (Thermo Fisher Scientific, Inc.) according to the manufacturer's protocol. The 

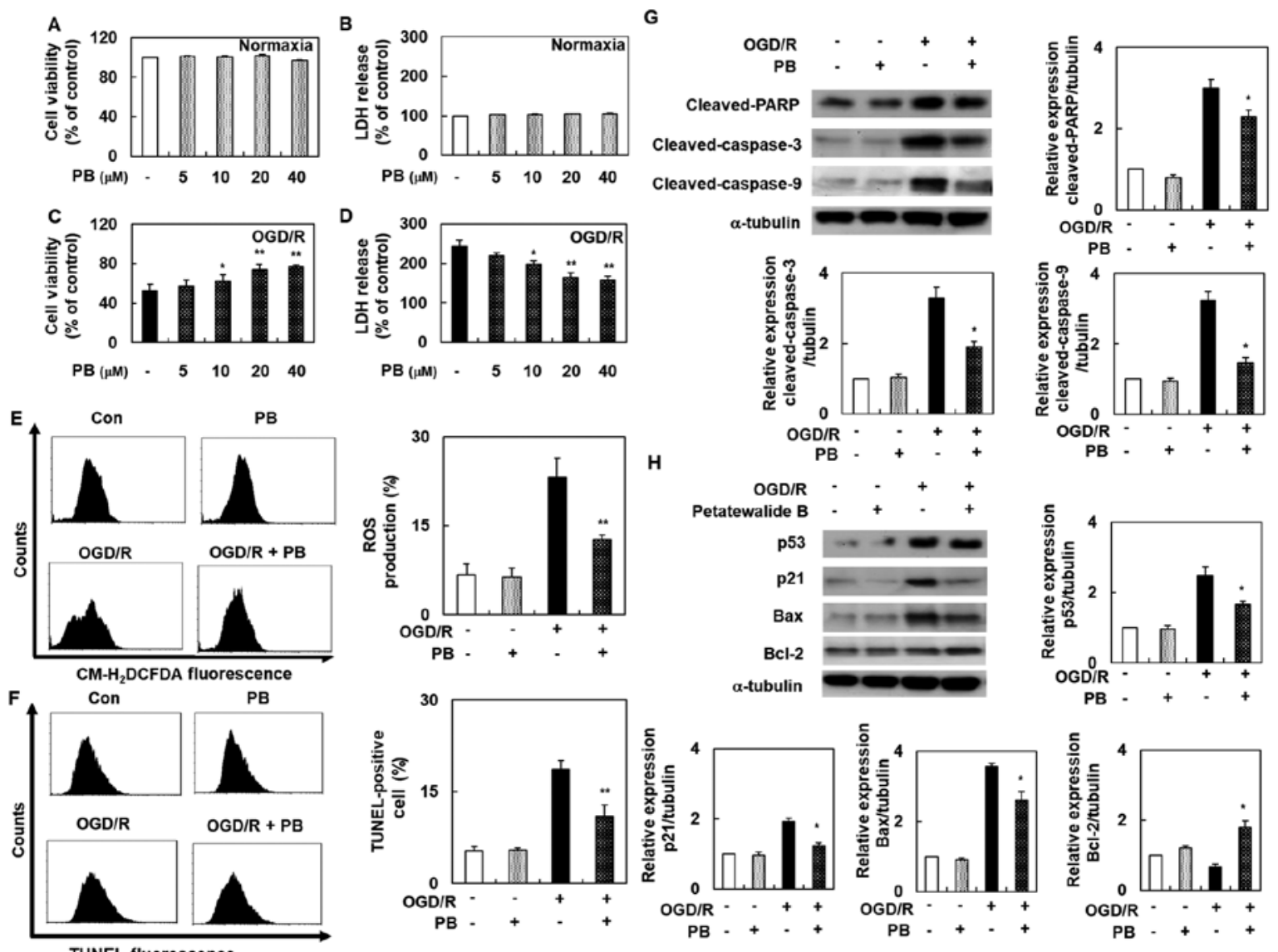

Figure 1. Cell death, ROS production and apoptosis in SH-SY5Y cells after OGD/R, with or without PB treatment. (A) MTT assays were used to examine the viability of SH-SY5Y cells treated with PB. (B) LDH assays were used to examine the cytotoxicity of SH-SY5Y cells treated with PB. (C) MTT assays were used to examine the viability of SH-SY5Y cells pretreated with PB and co-treated with OGD/R. (D) LDH assays were used to examine the cytotoxicity of SH-SY5Y cells pretreated with PB and co-treated with OGD/R. In an intracellular ROS assay, TUNEL staining and western blotting, cells were pretreated with PB $(20 \mu \mathrm{M})$ for $1 \mathrm{~h}$, followed by OGD/R for $8 \mathrm{~h}$ and then reoxygenated for a further $24 \mathrm{~h}$. (E) Representative images of ROS production measured by flow cytometry. (F) Demonstrative images of TUNEL-staining level measured by flow cytometry. (G) Western blot analysis was used to distinguish the protein expression levels of cleaved PARP, cleaved caspase-9 and cleaved caspase-3. (H) The protein expression levels of p53, Bax, and p21 were detected via western blotting. The values are presented as the mean $\pm \mathrm{SD}(\mathrm{n}=3)$. ${ }^{*} \mathrm{P}<0.05$ and ${ }^{* *} \mathrm{P}<0.01$ vs. OGD/R group. Con, control; LDH, lactate dehydrogenase; OGD/R, oxygen-glucose deprivation/reoxygenation; $\mathrm{PB}$, petatewalide $\mathrm{B}$; ROS, reactive oxygen species.

protein concentration was evaluated using the Bio-Rad protein assay kit (Bio-Rad Laboratories, Inc.).

Western blot analysis. The protein levels of cleaved caspase-3, cleaved caspase-9, cleaved PARP, p21, p53, Bax, Bcl-2, HO-1, NQO1, Nrf2, AMPK, GSK-3 $\beta$, p-AMPK, and p-GSK-3 $\beta$ in the SH-SY5Y cells were verified via western blot analysis. Equal quantities of protein samples resolved on $7.5-10 \%$ acrylamide sodium dodecyl sulfate-polyacrylamide gel electrophoresis (SDS-PAGE) gels were transferred to polyvinylidene fluoride (PVDF) membranes. The antibody dilution ratios were as follows: $\alpha$-tubulin (1:2,000), TBP (1:500), Nrf2 (1:500), NQO1 (1:500), and HO-1 (1:1,000; Santa Cruz Biotechnology, Inc.); cleaved caspase-3 $(1: 1,000)$, cleaved PARP $(1: 1,000)$, cleaved caspase-9 (1:1,000), p53 (1:1,000), p21 (1:1,000), Bax (1:1,000), AMPK $(1: 1,000)$, p-AMPK $(1: 1,000)$, GSK-3 $\beta(1: 1,000)$, and p-GSK-3 $\beta$ (1:1,000; Cell Signaling Technology Inc.). Thereafter, the membranes were incubated with a 1:5,000 dilution of goat anti-rabbit IgG-HRP secondary antibody (Cell Signaling Technology Inc.). Data were scanned using an imaging system (Amersham Biosciences) and analyzed using the ImageQuant 350 analyzer.
Small interfering RNA (siRNA) transfection. The knockdown of Nrf2, HO-1, and NQO1 in the SH-SY5Y cells was performed using a commercially available siRNA (Santa Cruz Biotechnology, Inc.). The cells were transfected using X-treme GENE siRNA Transfection Reagent (Roche Diagnostics), following the manufacturer's instructions.

Luciferase activity assay. ARE reporter plasmid assay was performed, as described in the FuGENE-HD transfection reagent manufacturer's protocol. Briefly, the cells were transfected with the ARE reporter plasmid diluted in Opti-MEM (Gibco; Thermo Fisher Scientific, Inc.) using FuGENE-HD reagent. The cells were gathered and lysed after transfection, and luciferase activity was verified using the Dual-Glo ${ }^{\circledR}$ Luciferase Assay System (Promega Corporation) with a luminometer (Victor3; Perkin-Elmer).

Statistical analysis. Statistical analyses were performed using SPSS 18.0 software (SPSS, Inc.). The experiments were repeated at least three times. Statistically significant differences were assessed using one-way analysis of variance (ANOVA) followed by Tukey's post-hoc test. Data are shown as 

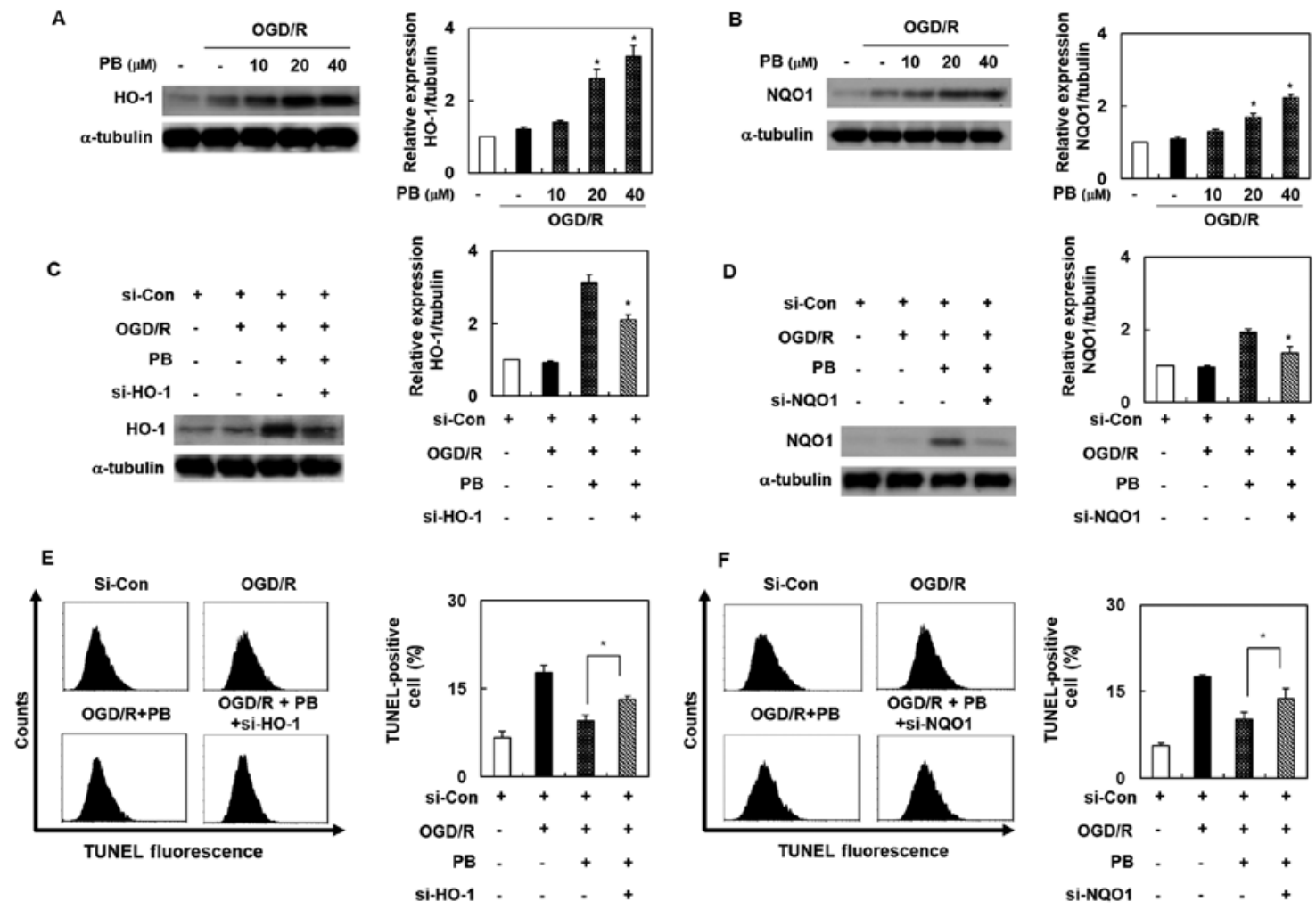

Figure 2. Expression levels of HO-1 and NQO1 in SH-SY5Y cells after OGD/R, with or without PB treatment. The relative protein expression levels of (A) HO-1 and (B) NQO1 were detected by western blot analysis (C) Cells were pretreated with PB $(20 \mu \mathrm{M})$, followed by OGD/R. Western blot analysis was used to verify the si-HO-1 transfection effects. (D) Western blot analysis was used to verify the si-NQO1 transfection effects. (E) Effect of si-HO-1-transfection on the TUNEL staining level in cells after OGD/R, with or without PB treatment $(20 \mu \mathrm{M})$. (F) Effect of si-NQO1 transfection on the TUNE-staining level in cells after OGD/R, with or without PB treatment $(20 \mu \mathrm{M})$. Values are presented as the mean $\pm \mathrm{SD}(\mathrm{n}=3)$. " $\mathrm{P}<0.05$ vs. OGD/R group. Con, control; HO-1, heme oxygenase 1; NQO1, NAD(P)H quinone dehydrogenase 1; OGD/R, oxygen-glucose deprivation/reoxygenation; PB, petatewalide B; si, small interfering RNA.

the mean \pm standard deviation $(\mathrm{SD})$. A $\mathrm{P}<0.01$ or $\mathrm{P}<0.05$ were considered to indicate a statistically significant difference.

\section{Results}

Petatewalide B ameliorates the OGD/R-exposed SH-SY5Y cell injury. Before evaluating petatewalide B's role, cell viability and cytotoxicity were investigated using the MTT and LDH assays. The results showed that petatewalide $\mathrm{B}$ concentrations up to $40 \mu \mathrm{M}$ did not alter cell viability and cytotoxicity. Our experimental goal was to examine petatewalide B's effect on I/R injury by constructing an OGD/R-induced SH-SY5Y cell model. In cell viability and cytotoxicity analysis, OGD/R-exposed SH-SY5Y cells exhibited a significant downregulation of cell viability and marked upregulation of cell cytotoxicity. However, these effects were eliminated by petatewalide B application. The high-dose group $(40 \mu \mathrm{M})$ exhibited much better effects than the low-dose group $(5 \mu \mathrm{M})$ (Fig. 1A-D). Since ROS is an important indicator of oxidative stress and neuronal injury, the level of ROS in the SH-SY5Y cells was determined. The results indicated that, compared with the control group, the ROS production level was markedly higher after OGD/R in the experimental group. However, treatment with petatewalide $\mathrm{B}$ remarkably reduced ROS production levels (Fig. 1E). We further examined the TUNEL-staining levels in each group because DNA fragments play a central role in apoptosis. Importantly, DNA fragment levels are elevated after OGD/R. Therefore, an increase in TUNEL-staining levels indicated that apoptosis was induced. The TUNEL-staining levels were remarkably higher in the $\mathrm{OGD} / \mathrm{R}$ group than in the control group. However, petatewalide B could inhibit the increase in TUNEL-staining levels after OGD/R, indicating that petatewalide B prevents apoptosis in OGD/R-exposed SH-SY5Y cells (Fig. 1F). Western blotting was performed to analyze gene expression downstream of apoptosis. OGD/R led to notable increases pro-apoptosis gene expressions (cleaved PARP, cleaved caspase-9, cleaved caspase-3, p53, p21, and Bax) and decreases in anti-apoptotic protein Bcl-2 expression in cells, compared to the control cells. As expected, genes related to pro-apoptosis and anti-apoptosis were significantly eliminated in the $\mathrm{OGD} / \mathrm{R}$ + petatewalide $\mathrm{B}$, compared to the $\mathrm{OGD} / \mathrm{R}$ group (Fig. $1 \mathrm{G}$ and $\mathrm{H}$ ). This indicated that petatewalide $\mathrm{B}$ may function as an inhibitor of apoptosis-related genes.

Petatewalide B-mediated inhibition of neuronal injury is inhibited by HO-1 and NQOI knockdown in OGD/R-induced SH-SY5Y cells. To elucidate the molecular mechanism by which petatewalide B induced neuroprotection, our experiment's first goal was to evaluate petatewalide B's effect on HO-1 and NQO1 expression in the SH-SY5Y cells exposed to $\mathrm{OGD} / \mathrm{R}$. The results indicated that petatewalide $\mathrm{B}$ elevated the protein levels of HO-1 and NQO1 (Fig. 2A and B). To confirm that the protective regulators, $\mathrm{HO}-1$ and NQO1, are responsible for the neuroprotective properties of petatewalide B, the cells were transfected with si-HO-1 or si-NQO1 and then administered petatewalide $\mathrm{B}$, followed by exposure to 

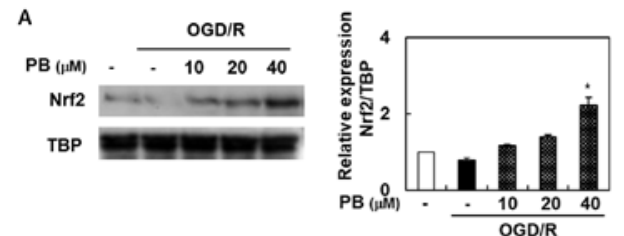

B
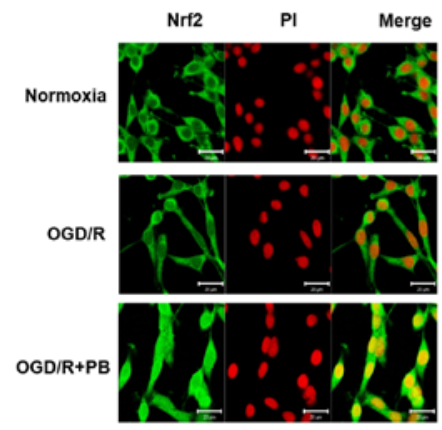

C

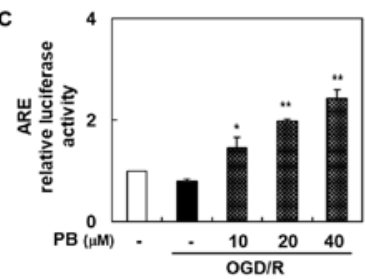

D
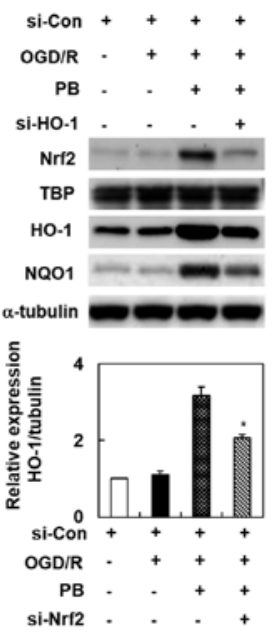

E

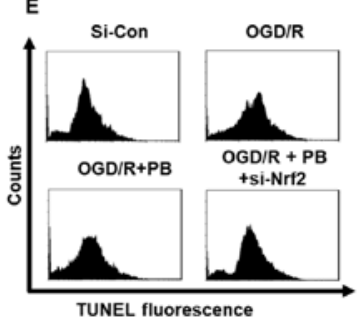

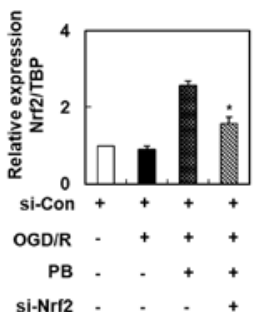

si-Nrf2 - . .
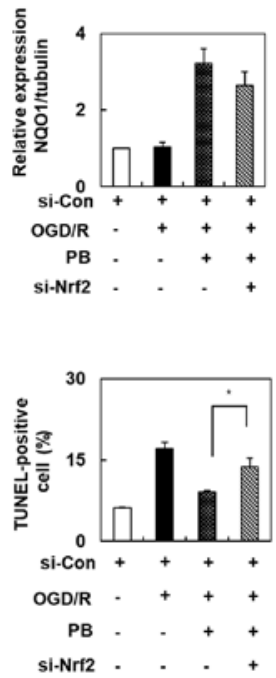

Figure 3. Activation of Nrf2 in SH-SY5Y cells after OGD/R, with or without PB treatment. (A) Nuclear Nrf2 levels were determined by western blotting. (B) Cells were pretreated with PB $(20 \mu \mathrm{M})$ and then exposed to OGD/R. Nrf2 distribution in SH-SY5Y cells was observed by immunofluorescence double-staining of Nrf2 (green) and PI (red). Scale bar, $20 \mu \mathrm{m}$. (C) ARE promoter activity was assessed using the dual-luciferase assay. (D) Cells were pretreated with PB $(20 \mu \mathrm{M})$, followed by OGD/R. Western blotting was used to verify the effects of si-Nrf2 transfection. (E) Effect of si-Nrf2 transfection on the TUNEL-staining level in cells after OGD/R, with or without $\mathrm{PB}$ treatment $(20 \mu \mathrm{M})$. The values are presented as the mean $\pm \mathrm{SD}(\mathrm{n}=3)$. ${ }^{*} \mathrm{P}<0.05$ and ${ }^{* * *} \mathrm{P}<0.01$ vs. OGD/R group. ARE, antioxidant response element; Con, control; HO-1, heme oxygenase 1; NQO1, NAD(P)H quinone dehydrogenase 1; Nrf2, nuclear factor erythroid 2-related factor 2; OGD/R, oxygen-glucose deprivation/reoxygenation; PB, petatewalide B; PI, propidium iodide; si, small interfering RNA

OGD/R. The results showed that the petatewalide B-induced expression of HO-1 and NQO1 was blocked by si-HO-1 or si-NQO1 (Fig. 2C and D). Furthermore, the increase of TUNEL-staining levels, induced by OGD/R, was significantly eliminated by petatewalide B; however, knockdown of HO-1 and NQO1 significantly attenuated petatewalide B's effects (Fig. 2E and F). In addition, knockdown of HO-1 and NQO1 could significantly reverse the upregulation of cell viability, as well as the downregulation of cell cytotoxicity, exerted by petatewalide B (data not shown). These results showed that petatewalide $\mathrm{B}$ could inhibit the neurotoxic response by inducing HO-1 and NQO1 expression.

Petatewalide B ameliorates neurotoxicity via the Nrf2/ARE signaling pathway in OGD/R-exposed $S H$-SY5Y cells. Our experiment's second goal was to determine Nrf2/ARE's role in petatewalide B's neuroprotective properties. We assessed the Nrf2/ARE signaling pathway activation by different means, including immunofluorescence staining, promoter assays, Western blotting, and siRNA transfection. The nuclear Nrf2 levels were evaluated using Western blot analysis to unravel transcriptional factor Nrf2's potential role following petatewalide B treatment. Nuclear Nrf2 levels were markedly higher in the OGD/R + petatewalide B group than in the OGD/R group (Fig. 3A). We further investigated nuclear Nrf2 distribution in SH-SY5Y cells using immunofluorescence double-staining of Nrf2 and PI. Petatewalide B increased the level of Nrf2 in the OGD/R-exposed cells, particularly in the nuclei (Fig. 3B). The target ARE sites of Nrf2 binding to the corresponding HO-1 and NQO1 were evaluated using the luciferase activity assay. The luciferase activity of cells transfected with the ARE reporter plasmids showed that petatewalide B elevated luciferase activity after OGD/R (Fig. 3C). Although it was demonstrated that petatewalide B could activate the Nrf2/ARE signal, it is still unknown whether the Nrf2/ARE signal was involved in petatewalide B's neuroprotective effects in the OGD/R-induced SH-SY5Y cells. Therefore, SH-SY5Y cells were transfected with si-Nrf2 and then treated with petatewalide B, followed by OGD/R. Thereafter, HO-1 and NQO1 expression in the si-Nrf2 + petatewalide B group was downregulated compared to the si-con + petatewalide B group (Fig. 3D). Furthermore, compared to the OGD/R group, the TUNEL-staining levels of the SH-SY5Y cells in the OGD/R + petatewalide B decreased. The TUNEL-staining levels of the SH-SY5Y cells in the si-Nrf2 group were higher than those of the si-con group cells in the OGD/R + petatewalide B group (Fig. 3E).

Petatewalide $B$ inhibits $O G D / R$-induced neurotoxicity by activating the Nrf2/ARE signal through AMPK. To evaluate the possibility that the AMPK signal was involved in petatewalide B's neuroprotective effect after OGD/R, the SH-SY5Y cells were treated with petatewalide B at different concentrations. Thereafter, the cells were collected for 
A

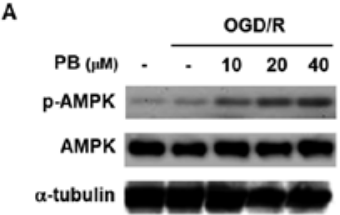

B

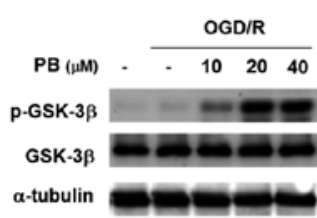

D

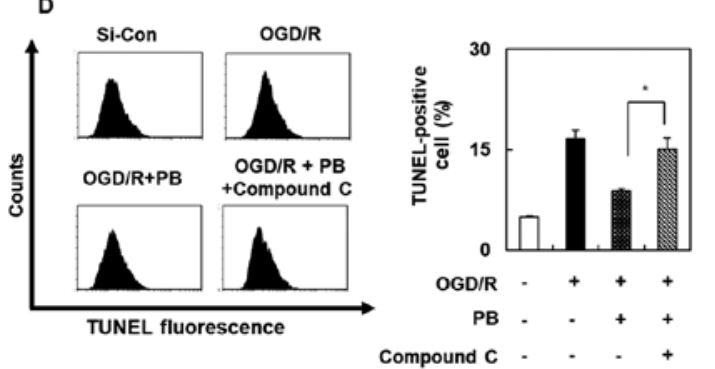

c
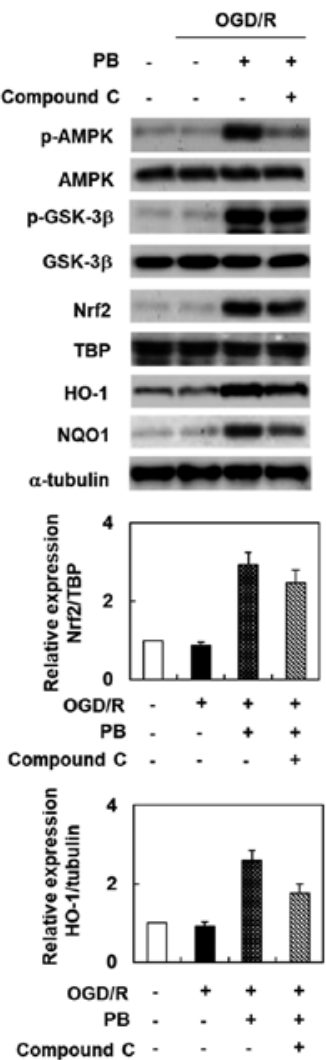

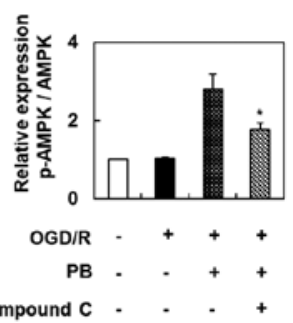

Compound C - - $\cdot$

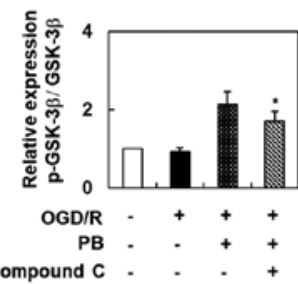

Compound $\mathrm{C}-1+$

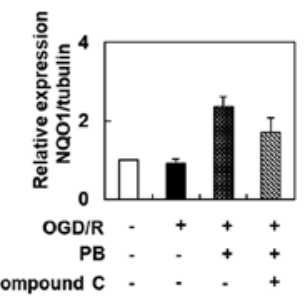

Figure 4. Activation of AMPK in SH-SY5Y cells after OGD/R, with or without PB treatment. (A) Phosphorylation levels of AMPK and (B) GSK-3 were determined by western blot analysis after OGD/R in SH-SY5Y cells, with or without PB pretreatment. (C) Cells were pretreated with PB (20 $\mu \mathrm{M})$, followed by OGD/R. Western blotting data revealing the relative level of p-AMPK, p-GSK-3, Nrf2, HO-1 and NQO1 from the control, OGD/R group, the group subjected to OGD/R and PB, and the group exposed to OGD/R, PB and compound C. (D) Cells were pretreated with PB (20 $\mu \mathrm{M}$ ) or compound C (10 $\mu \mathrm{M}$ ), followed by OGD/R. The effect of compound $\mathrm{C}$ on TUNEL-staining levels in the cells after OGD/R, with or without PB treatment. The values are presented as the mean $\pm \mathrm{SD}(\mathrm{n}=3)$. "P<0.05 vs. OGD/R group. AMPK, 5 ' AMP-activated protein kinase; Con, control; HO-1, heme oxygenase 1; GSK-3, glycogen synthase kinase 3; NQO1, NAD(P)H quinone dehydrogenase 1; Nrf2, nuclear factor erythroid 2-related factor 2; OGD/R, oxygen-glucose deprivation/reoxygenation; p-, phosphorylated; PB, petatewalide B; si, small interfering RNA

Western blotting analysis. The results indicated that phosphorylation of AMPK and GSK-3 $\beta$ was upregulated in the $\mathrm{OGD} / \mathrm{R}$ group, compared to the $\mathrm{OGD} / \mathrm{R}+$ petatewalide $\mathrm{B}$ group (Fig. 4A and B). Furthermore, petatewalide B could inhibit OGD/R-induced neurotoxicity by promoting AMPK activation. Compound $\mathrm{C}$ treatment (an AMPK inhibitor) was used to measure the activation of AMPK and downstream proteins, including GSK-3 $\beta$, Nrf2, HO-1, and NQO1. Petatewalide B-induced the phosphorylation of AMPK and GSK-3 $\beta$ and accumulation of nuclear Nrf2, as well as the expression of HO-1 and NQO1 in OGD/R; however, treatment with compound $\mathrm{C}$ significantly reversed these effects (Fig. 4C). Furthermore, compared with the group exposed to OGD/R + petatewalide B, the TUNEL-staining levels were further elevated by suppressing the Nrf2/ARE signal with compound $\mathrm{C}$ (Fig. 4D). These results indicate that AMPK inactivation partially abrogates petatewalide B's neuroprotective properties after OGD/R, which means that AMPK is partially responsible for the Nrf2/ARE activation elicited by petatewalide $\mathrm{B}$.

\section{Discussion}

Neuronal injuries frequently occur as I/R-induced injuries; thus, they are leading causes of neurodegenerative diseases (17).
Several studies assessed petatewalide B's pharmacological activity; however, the presence/absence of its neuroprotective effects on OGD/R-exposed SH-SY5Y cells is still unknown. In this study, we investigated petatewalide B's influence on cell viability, cytotoxicity, oxidative stress, and apoptosis in a model of human neuroblastoma SH-SY5Y cell injury induced by $\mathrm{OGD} / \mathrm{R}$. Our results verified that petatewalide $\mathrm{B}$ could eliminate OGD/R-induced cell viability, cytotoxicity, oxidative stress, and apoptosis by activating the AMPK/Nr2 signaling pathway, thereby protecting human neuroblastoma SH-SY5Y cells against OGD/R injury.

Neuronal apoptosis frequently occurs in neurodegenerative diseases, resulting in long-term alterations in brain function. Evidence suggests that excessive oxidative stress occurring in damaged neighboring neuronal cells could activate multiple cellular signaling pathways that lead to apoptosis, including DNA fragmentation, as well as genes related to pro-apoptosis and anti-apoptosis $(18,19)$. Here, we also demonstrated that petatewalide B could alleviate OGD/R-induced apoptosis, inhibit pro-apoptosis (cleaved PARP, cleaved caspase-9, cleaved caspase-3, p53, Bax, and p21), and increase the anti-apoptotic protein Bcl-2, demonstrating that this active constituent may exert its inhibitory effects on OGD/R-induced apoptosis by suppressing apoptosis-related genes. 
The pathological role of oxidative stress is demonstrated in a variety of central nervous system diseases, including ischemic, infectious, traumatic, and neurodegenerative diseases. In brain I/R injury, the return of blood supply may lead to excessive ROS-mediated oxidative stress damage, which could, subsequently, damage neighboring neuronal cells and even lead to neurodegenerative diseases $(2,4)$. Overall, oxidative stress is undoubtedly the effective intervention target for I/R injury. Due to its inhibitory effects on ROS production, petatewalide $\mathrm{B}$ exhibits a neuroprotective effect on OGD/R-injuries.

Neuronal injuries' pathophysiology are particularly complex. Thus, clarifying the underlying mechanisms contributes to the development of novel protective mechanisms. Nrf2 initiated the transcription of various cytoprotective genes involved in cell injury. It acted as a primary defensive molecule against neurotoxicity caused by several factors $(3,20)$. The Nrf2/ARE signaling pathway may be involved in one of petatewalide B's anti-neuroinflammation mechanisms (15). However, to our knowledge, no evidence of Nrf2's activation of petatewalide B on SH-SY5Y cells has been previously reported. In the present study, petatewalide B was demonstrated to protect SH-SY5Y cells from OGD/R-induced injury by activating the Nrf2/ARE signaling pathway; moreover, knockdown of Nrf2, HO-1, and NQO1 attenuated this protective effect. These results suggest that petatewalide $\mathrm{B}$ alleviated injury in OGD/R-exposed SH-SY5Y cells by activating the Nrf2/ARE signaling pathway.

Furthermore, the possible mechanisms of AMPK activation by petatewalide B were investigated. As mentioned earlier, the results of AMPK phosphorylation indicate that petatewalide B may have regulatory effects on neuroprotection in OGD/R-exposed SH-SY5Y cells because AMPK activation can reflect the degrees of neuroprotective responses. Previous studies have shown that the upstream signaling pathways involved in regulating the Nrf2/ARE signal mainly include MAPKs, AKT, and AMPK, among which AMPK activation can partially induce neuroprotective responses $(7,10)$. Our results showed that only the trend of Nrf2 activation was consistent with that of AMPK activation after treatment with petatewalide B. In addition, studies found that AMPK inhibitors can significantly inhibit neuroprotective properties by inactivating AMPK and downstream factors, including Nrf2, HO-1, and NQO1. Consistent with this finding, AMPK activation is responsible for the petatewalide $\mathrm{B}$-induced neuroprotective effects after OGD/R.

In summary, this study found that petatewalide B exerts neuroprotective effects on OGD/R-exposed SH-SY5Y cells by regulating the AMPK/Nrf2/ARE signaling pathway, which represents a new breakthrough in the treatment of I/R injury.

\section{Acknowledgements}

Not applicable.

\section{Funding}

The present study was supported by the Basic Science Research Program through the National Research Foundation of Korea, funded by the Ministry of Education (grant nos. NRF2018R1D1A1B07047825 and NRF-2018R1D1A3B07047983).

\section{Availability of data and materials}

All data generated or analyzed during this study are included in this published article.

\section{Authors' contributions}

SYP, MHC, ML, KL, GP and YWC designed and performed the experiments. SYP, MHC, GP and YWC analyzed and interpreted the data. SYP, MHC, ML and YWC prepared and revised the manuscript. All authors read and approved the final manuscript.

\section{Ethics approval and consent to participate}

Not applicable.

\section{Patient consent for publication}

Not applicable.

\section{Competing interests}

The authors declare that they have no competing interests.

\section{References}

1. Wang J, Wang A, He H, She X, He Y, Li S, Liu L, Luo T, Huang N, Luo $\mathrm{H}$, et al: Trametenolic acid B protects against cerebral ischemia and reperfusion injury through modulation of microRNA-10a and PI3K/Akt/mTOR signaling pathways. Biomed Pharmacother 112: 108692, 2019.

2. Dong RF, Tai LW, Zhang B, Shi FK, Liu HM, Duan PC and Cheng Y: Neuroprotective effect of FMS-like tyrosine kinase-3 silence on cerebral ischemia/reperfusion injury in a SH-SY5Y cell line. Gene 697: 152-158, 2019.

3. Hu S, Wu Y, Zhao B, Hu H, Zhu B, Sun Z, Li P and Du S: Panax notoginseng saponins protect cerebral microvascular endothelial cells against oxygen-glucose deprivation/reperfusion-induced barrier dysfunction via activation of PI3K/akt/Nrf2 antioxidant signaling pathway. Molecules 23: E2781, 2018.

4. Feng L, Gao J, Liu Y, Shi J and Gong Q: Icariside II alleviates oxygen-glucose deprivation and reoxygenation-induced PC12 cell oxidative injury by activating Nrf2/SIRT3 signaling pathway. Biomed Pharmacother 103: 9-17, 2018.

5. Sun X, Li X, Ma S, Guo Y and Li Y: MicroRNA-98-5p ameliorates oxygen-glucose deprivation/reoxygenation (OGD/R)-induced neuronal injury by inhibiting Bach1 and promoting Nrf2/ARE signaling. Biochem Biophys Res Commun 507: 114-121, 2018.

6. Li F, Liang J and Tang D: Brahma-related gene 1 ameliorates the neuronal apoptosis and oxidative stress induced by oxygen-glucose deprivation/reoxygenation through activation of Nrf2/HO-1 signaling. Biomed Pharmacother 108: 1216-1224, 2018.

7. Yin WL, Yin WG, Huang BS and Wu LX: LncRNA SNHG12 inhibits miR-199a to upregulate SIRT1 to attenuate cerebral ischemia/reperfusion injury through activating AMPK signaling pathway. Neurosci Lett 690: 188-195, 2019.

8. Ting-Ting L, Yuan G, Jun L, Dan X and Hong-Bo T: GSK621 attenuates oxygen glucose deprivation/re-oxygenation-induced myocardial cell injury via AMPK-dependent signaling. Biochem Biophys Res Commun 514: 826-834, 2019.

9. Duan Q, Sun W, Yuan H and Mu X: MicroRNA-135b-5p prevents oxygen-glucose deprivation and reoxygenation-induced neuronal injury through regulation of the GSK-3 $\beta / \mathrm{Nrf} 2 / \mathrm{ARE}$ signaling pathway. Arch Med Sci 14: 735-744, 2018.

10. Duan J, Cui J, Yang Z, Guo C, Cao J, Xi M, Weng Y, Yin Y, Wang Y, Wei G, et al: Neuroprotective effect of apelin 13 on ischemic stroke by activating AMPK/GSK-3beta/Nrf2 signaling. J Neuroinflammation 16: 24, 2019. 
11. Zhou F, Wang M, Ju J, Wang Y, Liu Z, Zhao X, Yan Y, Yan S, Luo $X$ and Fang Y: Schizandrin A protects against cerebral ischemia-reperfusion injury by suppressing inflammation and oxidative stress and regulating the AMPK/Nrf2 pathway regulation. Am J Transl Res 11: 199-209, 2019.

12. Adachi Y, Kanbayashi Y, Harata I, Ubagai R, Takimoto T, Suzuki K, Miwa T and Noguchi Y: Petasin activates AMP-activated protein kinase and modulates glucose metabolism. J Nat Prod 77: 1262-1269, 2014.

13. Choi YW, Lee KP, Kim JM, Kang S, Park SJ, Lee JM, Moon HR, Jung JH, Lee YG and Im DS: Petatewalide B, a novel compound from Petasites japonicus with anti-allergic activity. J Ethnopharmacol 178: 17-24, 2016

14. Kitajima M, Okabe K, Yoshida M, Nakabayashi R, Saito K, Kogure N and Takayama H: New otonecine-type pyrrolizidine alkaloid from Petasites japonicus. J Nat Med 73: 602-607, 2019.

15. Park SY, Choi MH,Li M,Li K, Park G and Choi YW: AMPK/Nrf2 signaling is involved in the anti-neuroinflammatory action of Petatewalide B from Petasites japonicus against lipopolysaccharides in microglia. Immunopharmacol Immunotoxicol 40 232-241, 2018.

16. Wang S, Jin DQ, Xie C, Wang H, Wang M, Xu J and Guo Y: Isolation, characterization, and neuroprotective activities of sesquiterpenes from Petasites japonicus. Food Chem 141: 2075-2082, 2013.
17. Yao X, Yao R, Yi J and Huang F: Upregulation of miR-496 decreases cerebral ischemia/reperfusion injury by negatively regulating BCL2L14. Neurosci Lett 696: 197-205, 2019.

18. Zeng Z, Zhang Y, Liang X, Wang F, Zhao J, Xu Z, Liu X and Liu X: Qingnao dripping pills mediate immune-inflammatory response and MAPK signaling pathway after acute ischemic stroke in rats. J Pharmacol Sci 139: 143-150, 2019.

19. Sun B, Ou H, Ren F, Huan Y,Zhong T, Gao M and Cai H: Propofol inhibited autophagy through $\mathrm{Ca}^{2+} / \mathrm{CaMKK} / \mathrm{AMPK} / \mathrm{mTOR}$ pathway in OGD/R-induced neuron injury. Mol Med 24: 58, 2018.

20. Young Park S, Jin Kim Y, Park G and Kim HH: Neuroprotective effect of Dictyopteris divaricata extract-capped gold nanoparticles against oxygen and glucose deprivation/reoxygenation. Colloids Surf B Biointerfaces 179: 421-428, 2019.

This work is licensed under a Creative Commons Attribution-NonCommercial-NoDerivatives 4.0 International (CC BY-NC-ND 4.0) License. 